\title{
J-PARC E34 g-2/EDM experiment
}

\section{Masashi Otani*}

High Energy Accelerator Research Organization (KEK), Tsukuba, Ibaraki 305-0801, Japan

E-mail: masashio@post.kek.jp

The muon anomalous magnetic moment, $a_{\mu}=\left(g_{\mu}-2\right) / 2$, was measured with a precision of $0.54 \mathrm{ppm}$ at Brookhaven National Laboratory (BNL) around 15 years ago. The measured value differs from the Standard Model (SM) prediction by more than three standard deviations. This may imply existence of new physics beyond SM. The new J-PARC experiment aims to measure $a_{\mu}$ and the electric dipole moment (EDM) by a completely different method to that at BNL. In the JPARC experiment, ultracold muons are stored in a $0.66 \mathrm{~m}$-diameter magnetic field without electric focusing. The ultracold muons are produced by laser-ionising muonium atoms accelerated by the muon-dedicated linear accelerator. This paper reports the current status of each experimental component.

XIV International Conference on Heavy Quarks and Leptons (HQL2018)

May 27- June 1, 2018

Yamagata Terrsa, Yamagata,Japan

\footnotetext{
${ }^{*}$ Speaker.
} 


\section{Introduction}

Though the discovery of Higgs at LHC completed a list of the particles predicted in Standard Model (SM) of elementary particle physics, some observations such as possible existence of dark matter indicate new physics beyond SM at some energy or interaction scale. One of the clues for new physics is the muon anomalous magnetic moment $a_{\mu}=\left(g_{\mu}-2\right) / 2$; it has been measured to $0.54 \mathrm{ppm}$ [1] and a discrepancy of more than 3 standard deviations between measurement and the SM prediction was observed. This anomaly stimulated theorists to performing challenging calculations of corrections and uncertainties in the SM prediction of $a_{\mu}$. After many years of scrutiny, the discrepancy remains unexplained [2]. Along with the theoretical efforts, new experiments should address this anomaly.

The previous experiment at Brookhaven National Laboratory (BNL), E821, used the technique so called magic momentum. Because the muon beam generated from the secondary pions has large emittance, strong electric focusing in addition to the magnetic field is necessary in a storage ring. The difference of the cyclotron motion frequency $\left(\vec{\omega}_{c}\right)$ and the muon spin precession frequency $\left(\vec{\omega}_{s}\right)$ is given by

$$
\vec{\omega}_{a}=\vec{\omega}_{s}-\vec{\omega}_{c}=-\frac{e}{m}\left[a_{\mu} \vec{B}-\left(a_{\mu}-\frac{1}{\gamma^{2}-1}\right) \frac{\vec{\beta} \times \vec{E}}{c}+\frac{\eta}{2}\left(\vec{\beta} \times \vec{B}+\frac{\vec{E}}{c}\right)\right]
$$

where $e$ is elementary charge, $m$ is muon mass, $a_{\mu}$ is anomalous magnetic moment, $\gamma$ is the Lorentz factor, $\beta$ is the ratio of particle velocity to the speed of light $c$, and $\eta$ is electric dipole moment. The second term depending on the electric field is eliminated when the muon momentum is 3.094 $\mathrm{GeV} / \mathrm{c}$, so called magic momentum. Measurement using a new method will verify the $a_{\mu}$ anomaly.

The muon electric dipole moment (EDM) is also highly sensitive to new physics because it is strongly suppressed in SM $\left(10^{-38} e \cdot \mathrm{cm}\right)$, and violates CP symmetry assuming the CPT theorem. In addition to that, there is a possibility that the $a_{\mu}$ anomaly measured by the BNL E821 experiment can be explained by finite EDM with an order of $10^{-20} e \cdot \mathrm{cm}$ [3], whereas the current direct limit is $1.9 \times 10^{-19} e \cdot \mathrm{cm}[4]$.

The J-PARC E34 experiment [5] aims to measure $a_{\mu}$ with a precision of $0.1 \mathrm{ppm}$ and search for EDM with a sensitivity of about $10^{-21} e \cdot \mathrm{cm}$ by utilizing a high intensity beam and newly developed technique of the ultracold muon beam. Figure 1 shows the experimental setup. The experiment utilizes the proton beam from the $3 \mathrm{GeV}$ synchrotron ring to Material and Life Science Experiment Facility (MLF). The proton beam is injected to the graphite target. The generated surface muons are extracted to one of the muon beamline, H-line. The surface muons stop in the silica aerogel and approximately half of the stopped muons form thermal muoniums $\left(\mu^{+} e^{-}\right)$. The paired electron in the muonium is knocked out by a laser and thermal muon $(3 \mathrm{keV} / \mathrm{c})$ is generated. Then the muon is accelerated up to $300 \mathrm{MeV} / \mathrm{c}$ and injected to the storage ring supplying $3 \mathrm{~T}$. The decay positron is detected by a silicon strip tracker and the spin precession frequency is obtained from variation of counting rate of the decay positron. Thanks to the ultracold beam $\left(\sigma_{p T} / p=10^{-5}\right)$ where $p_{T}$ is the transverse momentum of the beam particles, the electric focusing is not necessary anymore. Eq. 1.1 becomes

$$
\vec{\omega}_{a}=-\frac{e}{m}\left[a_{\mu} \vec{B}+\frac{\eta}{2}(\vec{\beta} \times \vec{B})\right]
$$


The anomalous magnetic moment and EDM are perpendicular to each other. Therefore these can be measured simultaneously.

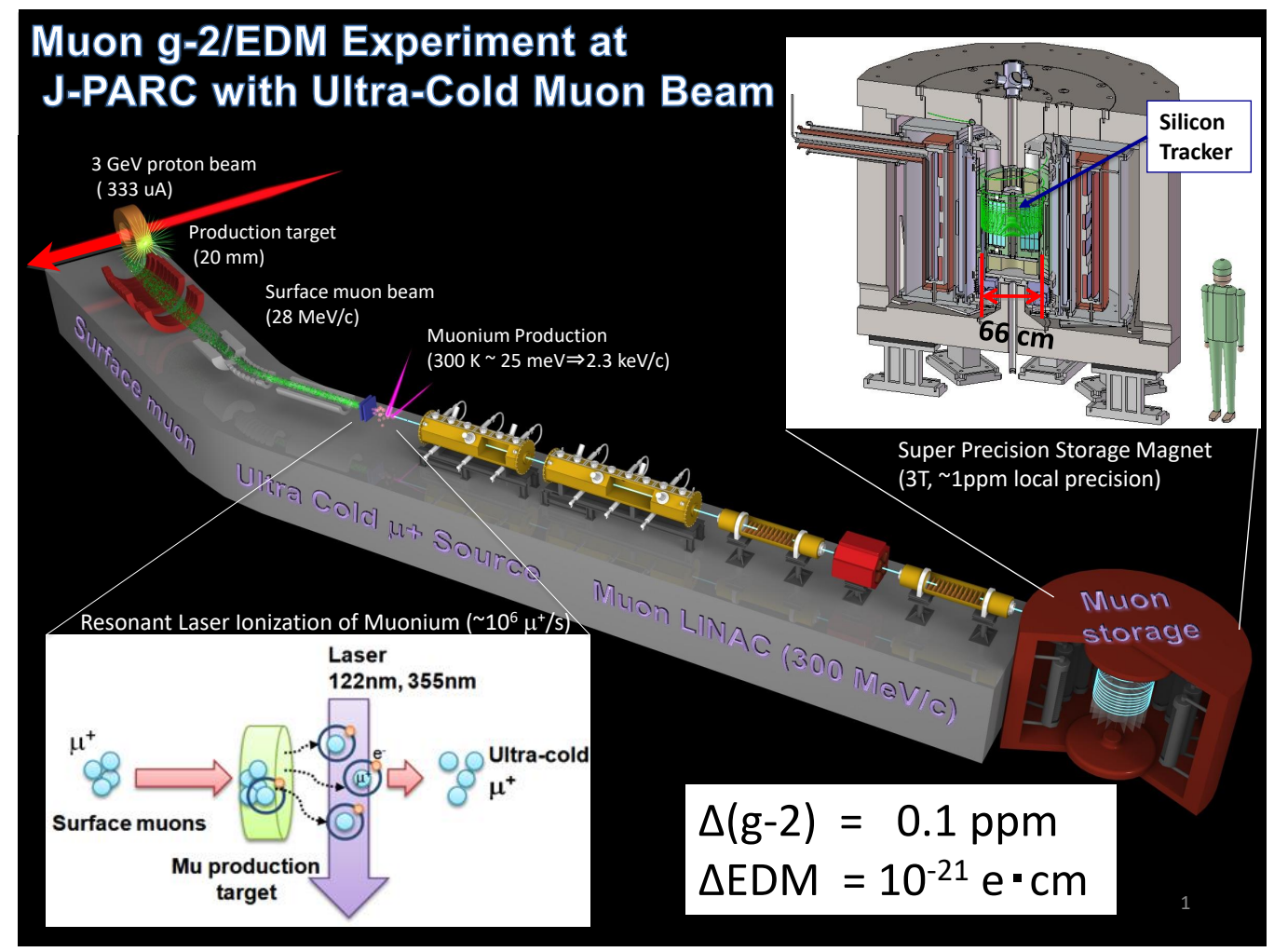

Figure 1: Overview of the J-PARC E34 experiment.

\section{Components of the J-PARC experiment}

\subsection{J-PARC accelerator}

The J-PARC accelerator is comprised of the $400-\mathrm{MeV} \mathrm{H}^{-}$linac, 3-GeV rapid synchrocyclotron (RCS), and $30-\mathrm{GeV}$ main ring. The MLF uses a $3 \mathrm{GeV}$ proton beam from RCS to produce muons and neutrons. The primary goal of the proton beam intensity at MLF is $1 \mathrm{MW}$, on the basis of which our statistical reach for $a_{\mu}$ and muon EDM is estimated. Presently, the J-PARC linac provides beams with a peak current of $40 \mathrm{~mA}$ and RCS provides a $500-\mathrm{kW}$ beam power to MLF. The peak current in the J-PARC linac will be upgraded to 50mA from Oct. 2018, that is necessary to provide the 1-MW beam power to MLF. RCS has successfully demonstrated 1-MW beam operation for one hour in summer 2018. The beam power at MLF is scheduled to be gradually increased to $1 \mathrm{MW}$ for use by experiments.

\subsection{Thermal muon production}

The ultracold muons are generated by laser-ionizing muonium. The first step is to produce the muoniums in a muonium production target. Silica aerogel is chosen as the muonium production 
target because it can be used at the room temperature and energy of the generated muons satisfies our requirement $(\mathrm{p}=3 \mathrm{keV} / \mathrm{c})$. Our measurements with silica aerogel [8] reveal that the diffusion length of muonium in the silica aerogel is $30 \mu \mathrm{m}$ which is much shorter than the muon stopping distribution of several millimeters. On the basis of the measurement, the silica aerogel with submillimeter structure was fabricated by using a femtometer laser (Fig. 2). Figure 3 shows the timing distribution of the reconstructed decay positrons downstream of the laser-ablated aerogel with equal spacing of $300 \mu \mathrm{m}$ in comparison to that from the silica aerogel without ablation. The data are consistent with the assumption of emission from the aerogel following diffusion within it and to the ablation holes with speeds corresponding to thermal velocity. The muonium emission was enhanced by approximately one order of magnitude.

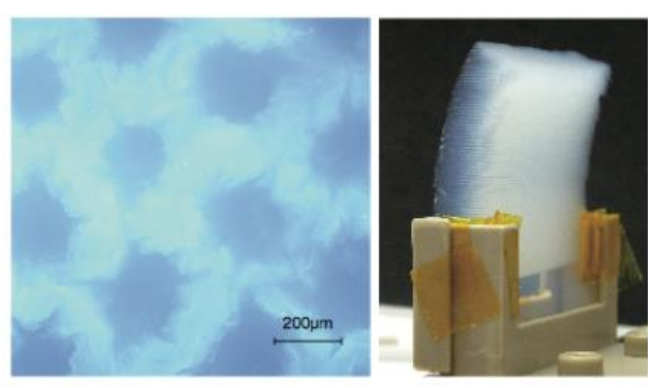

Figure 2: (Left) Photo of surface on the laserablated aerogel target. (Right) Whole picture of the target. The muon beam enters from the left and muonium is exiting from the right surface. Ref. [9]

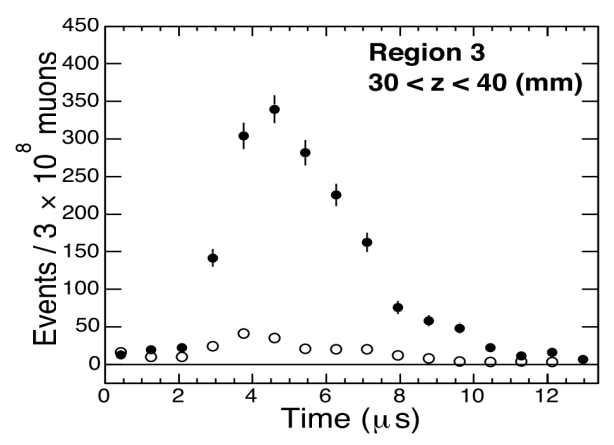

Figure 3: Time distribution of positrons in near the target surface for flat aerogel (open circles) and laser-ablated aerogel (close circles). No background has been subtracted. Ref. [9].

A high-power ionizing laser synchronized to the periodic $25 \mathrm{~Hz}$ muon beam is employed. The maximum density occurs approximately $1 \mu$ s after muons arrive to the aerogel. One laser at $122 \mathrm{~nm}$ excites the muonium from the $1 \mathrm{~s}$ atomic state to $2 \mathrm{p}$, then a second at $355 \mathrm{~nm}$ strips the electron. The laser ionization efficiency is based on a calculation assuming individual rates of induced emission and absorption as well as spontaneous photon emission [10]. The ionization efficiency is 0.73 for $100 \mathrm{~mJ}$ of Lyman- $\alpha$ ( $1 \mathrm{~S}$ to $2 \mathrm{~S}$ ) radiation and $300 \mathrm{~mJ}$ ionization ( $2 \mathrm{~S}$ to continuum) radiation.

\subsection{Muon acceleration}

Because muon has a finite lifetime, the muon should be accelerated in a sufficiently short period of time to suppress the decay loss. To realize fast acceleration, a muon-dedicated linear accelerator (linac) is developed. Since velocity $(\beta)$ of a muon largely varies during acceleration, several types of radiofrequency (RF) cavities should be adopted to realize sufficiently effective acceleration along with $\beta$. Figure 4 shows configuration of the muon linac. There are four kinds of the RF acceleration cavity: radio-frequency quadrupole linac (RFQ) [11], inter-digital H-mode drift tube linac (IH-DTL) [12], disk and washer coupled cell linac (DAW CCL) [13], and disk-loaded structure [14].

The muon linac is an unproven technology, and thus muon acceleration should be demonstrated prior to construction of the muon linac. We performed muon acceleration using a prototype 


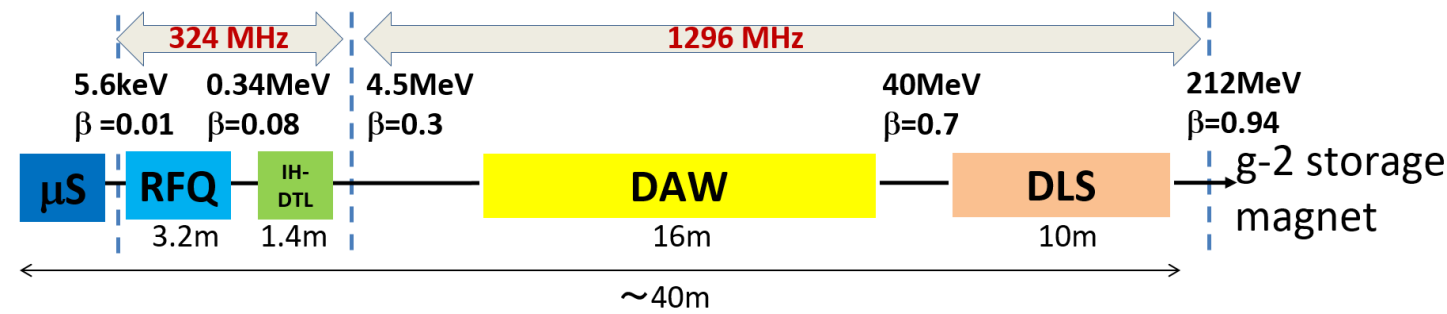

Figure 4: Configuration of the muon linac

of the RFQ in October 2017 at the J-PARC MLF test muon beamline. In the experiment, negative muonium ions $\left(\mathrm{Mu}^{-}\right.$'s, $\left.\mu^{+} e^{-} e^{-}\right)$are generated from $\mu^{+}$'s through the electron capture process in an aluminum foil. The generated $\mathrm{Mu}^{-}$'s are initially electrostatically accelerated and injected into a prototype RFQ. In the RFQ, the $\mathrm{Mu}^{-}$'s are accelerated to $89 \mathrm{keV}$. The accelerated $\mathrm{Mu}^{-}$'s are identified by measuring the momentum and time of flight. The measured time of flight was consistent with that expected from the accelerated $\mathrm{Mu}^{-}$'s. It is concluded that the muons have been accelerated by a radio-frequency accelerator for the first time [15].

\subsection{Injection and storage magnet}

Because the radius of the beam orbit in the storage magnet is only $33.3 \mathrm{~cm}$, the same apparatus as in the previous experiment, a device called an inflector and kicker, can not be used in our experiment. That is why a three-dimensional spiral injection scheme is adopted. The muons pass in a downward direction through a small entrance hole in the upper steel yoke of the storage magnet. Once inside the storage magnet, the muons go to the storage region after adjusting the remaining vertical momentum by a pulsed radial magnetic field. In order to match the acceptance of the spiral injection, the transport beamline after the muon linac was designed [16].

Figure 5 shows the storage magnet design. The storage magnet consists of four superconducting coils supplying the injection field, focusing field and main field of $3 \mathrm{~T}$ with local uniformity of $1 \mathrm{ppm}$. In order to achieve high homogeneity of the magnetic field below $1 \mathrm{ppm}$, the error field is corrected by shimming with iron pieces inside the magnet bore and superconducting shimming coils [17].

\subsection{Detector}

The decay positron detector is required to be highly segmented and operated at $3 \mathrm{~T}$. To satisfy these requirements, the silicon strip detectors are radially placed in the detection volume to efficiently detect the circular track of the positrons (Fig. 6). Because the positron hit rate changes significantly during one interval after the muon beam pulse injection, control of the pileup effects is critical for the detector. The strip pitch was chosen to be $0.19 \mathrm{~mm}$ on the basis of the simulation study [18].

\section{Statistical precision}

All the experimental components are designed based on measurements or, if not possible, 


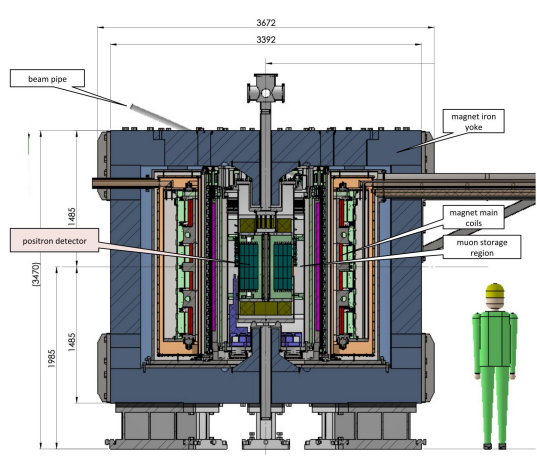

Figure 5: Section view of the muon storage magnet.

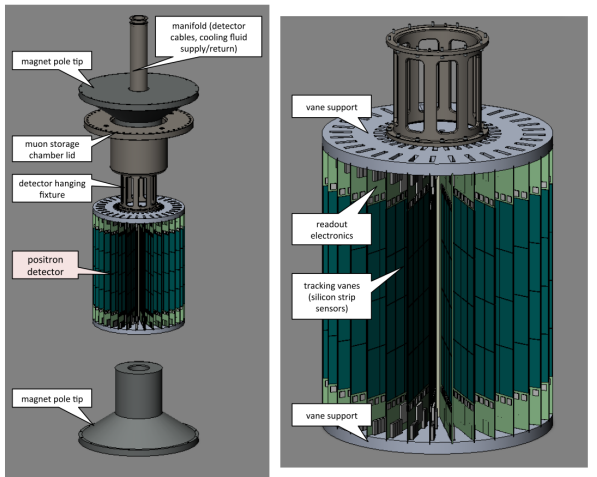

Figure 6: Schematic view of the positron tracking detector.

simulations. Table 1 summarizes our current estimates of the efficiencies of each step and number of muons. On the basis of this intensity estimation, the statistical uncertainty of the anomalous magnetic moment is 0.46 assuming a total running time of $2 \times 10^{7} \mathrm{~s}$ with a muon polarization of 0.5 . On the other hand, the EDM can be measured with a statistical uncertainty $\Delta d_{\mu}=1.4 \times 10^{-21} e \cdot \mathrm{cm}$.

Table 1: Estimated efficiency of each step and remaining muon beam intensity.

\begin{tabular}{lcl}
\hline Step & Efficiency & Intensity $(\mathrm{Hz})$ \\
\hline Muon intensity at pro- & & $2.0 \times 10^{9}$ \\
duction target & & \\
H-line transmission & 0.16 & $3.2 \times 10^{8}$ \\
Mu emission & $3.8 \times 10^{-3}$ & $1.2 \times 10^{6}$ \\
Laser ionization & 0.73 & $9.0 \times 10^{5}$ \\
Metal mesh & 0.78 & $7.0 \times 10^{5}$ \\
Electrostatic lens & 0.72 & $5.0 \times 10^{5}$ \\
RFQ transmission & 0.81 & $3.8 \times 10^{5}$ \\
IH transmission & 0.99 & $3.8 \times 10^{5}$ \\
IH decay & 0.99 & $3.8 \times 10^{5}$ \\
DAW transmission & 0.99 & $3.7 \times 10^{5}$ \\
DAW decay & 0.96 & $3.6 \times 10^{5}$ \\
DLS transmission & 1.00 & $3.6 \times 10^{5}$ \\
DLS decay & 0.99 & $3.5 \times 10^{5}$ \\
Injection transmission & 0.85 & $2.8 \times 10^{5}$ \\
Injection decay & 0.99 & $2.8 \times 10^{5}$ \\
Detector start time & 0.93 & $2.6 \times 10^{5}$ \\
\hline Muons at storage & & $2.6 \times 10^{5}$ \\
\hline
\end{tabular}




\section{Summary}

A precise measurement of $a_{\mu}$ is one of the promising paths to establish new physics beyond SM and muon EDM is sensitive to new physics because it is very suppressed in SM. E34 aims to measure $a_{\mu}$ and muon EDM with high precision and sensitivity with newly developed novel method of the ultracold muon beam. The statistical uncertainty for our Phase 1 measurement is estimated to be $0.46 \mathrm{ppm}$ and $1.4 \times 10^{-21} e \cdot \mathrm{cm}$ for $a_{\mu}$ and muon EDM, respectively.

\section{Acknowledgement}

This work is supported by JSPS KAKENHI Grant Numbers , JP15H03666, JP15H05742, JP16H03987, JP16J07784, and 18H03707.

\section{References}

[1] G.W. Bennett et al.: Phys. Rev. D 73 (2006) 072003

[2] A. Keshavarzi et al.: Phys. Rev. D 97 (2018) 114025

[3] J.L. Feng, K. T. Matchev and Y. Shadmi: Nucl. Phys. B 613 (2001) 366

[4] G.W. Bennett et al.: Phys. Rev. D 80 (2009) 052008

[5] http://g-2.kek.jp/portal/index.html

[6] A.P. Mills, Jr. et al.: Phys. Rev. Lett. 56 (1986) 1463

[7] P. Bakule et al.: Nucl. Instrum. Meth. B 266 (2008) 335-346

[8] P. Bakule et al.: Prog. Theor. Exp. Phys., 103 (2013) C01

[9] G.A. Beer et al.: Prog. Theor. Exp. Phys., 091 (2014) C01

[10] See, for example, H.A. Bethe, and E.E. Salpeter, "Quantum Mechanics of One- and Two-electrons Atoms", Sect. 68, Springer-Verlag, Berlin (1957).

[11] Y. Kondo et al., Phys. Rev. Accel. Beams 16 (2013) 040102

[12] M. Otani et al., Phys. Rev. Accel. Beams 19 (2016) 040101

[13] M. Otani et al., Proc. of IPAC2016, Busan, Korea, (2016) pp. 1543 - 1546

[14] Y. Kondo et al., Journal of Physics: Conference Series 874 (2017) 012054

[15] S. Bae et al., Phys. Rev. Accel. Beams 21 (2018) 050101

[16] H. Iinuma et al., Nucl. Instrum. Meth. A 832 (2016) 51-62

[17] M. Abe et al., Nucl. Instrum. Meth. A 890 (2018) 51-63

[18] S. Nishimura et al., JPS Conf. Proc. 8 (2015) 025015 